\title{
On the Convergence Properties of Quantum-Inspired Multi-Objective Evolutionary Algorithms
}

\author{
Zhiyong $\mathrm{Li}^{1}$, Zhe $\mathrm{Li}^{1}$, and Günter Rudolph ${ }^{2}$ \\ ${ }^{1}$ School of Computer and Communication, Hunan University \\ 410082 Changsha, Hunan, P.R.China \\ ${ }^{2}$ Fachbereich Information, Universität Dortmund \\ 44221 Dortmund, Germany
}

\begin{abstract}
In this paper, a general framework of quantum-inspired multiobjective evolutionary algorithms is proposed based on the basic principles of quantum computing and general schemes of multi-objective evolutionary algorithms. One of the sufficient convergence conditions to Pareto optimal set is presented and proved under partially order set theory. Moreover, two improved Q-gates are given as examples meeting this convergence condition.
\end{abstract}

Keywords: Quantum computing, multi-objective evolutionary algorithms, Pareto optimal set, stochastic convergence.

\section{Introduction}

Many optimization problems in scientific and engineering fields involve simultaneously two or more objectives that are competing or in conflict with each other frequently. They are known as multi-objective optimization problems (MOP). Ordinary MOPs have a set of optimal solutions, which is called Pareto solutions set. The plot of the objective functions whose vectors of the decision variables are in the Pareto solutions set is called the Pareto front[1].

As evolutionary algorithms have more advantage in dealing with discontinuous and concave Pareto fronts than traditional mathematical programming techniques, a large number of multi-objective evolutionary algorithms (MOEA) have been proposed during the last two decades, such as MOGA[2], NPGA[3], NSGA2[4], SPEA2[5] etc. Some important theoretical work related to MOEA has been done. Rudolph has investigated convergence properties of some MOEAs under partially ordered finite set theory[6, 7]. Hanne presented an evolutionary algorithm for approximating the efficient set of MOP [8].

Meanwhile, the quantum mechanical computational theory is attracting serious attention, some quantum-inspired evolutionary algorithms (QEA) were proposed in[911]. In last two years, some specific algorithms combining MOEA with QEA, which are called quantum-inspired multi-objective evolutionary algorithms (QMOEA) in this paper, were proposed $[12,13]$. Those experiments results show better proximity performance as well as diversity maintenance. However, few theoretical results on the QMOEA have been done.

D.-S. Huang, L. Heutte, and M. Loog (Eds.): ICIC 2007, CCIS 2, pp. 245-255, 2007.

(C) Springer-Verlag Berlin Heidelberg 2007 
In this paper, we will propose a general framework of QMOEA, and discuss its sufficient convergence conditions to the Pareto optimal set and give several example algorithms.

\section{Preliminaries}

MOP can be defined with a mathematical formulation as follows:

$$
\text { Optimum } f(\boldsymbol{x})=\left(f_{1}(\boldsymbol{x}), f_{2}(\boldsymbol{x}), \ldots f_{\mathrm{d}}(\boldsymbol{x})\right)^{\mathrm{T}} \quad \text { s.t. } \mathrm{x} \in \mathrm{S}
$$

where $f: \mathrm{S} \rightarrow \mathrm{R}^{d}$ is a vector-valued objective function, $\boldsymbol{x}=\left[x_{1}, x_{2}, \ldots, x_{k}\right]^{\boldsymbol{T}}$ is the vector of decision variables and the $S$ is the feasible set, which is usually defined by some constraint functions,

$$
S=\left\{x \in R^{k}: g_{i}(x) \leq 0, h_{j}(x)=0 ; i=1,2 \ldots p, j=1,2 \ldots q\right\} .
$$

Without loss of generality, let "optimum" mean "minimum" here. We say that a solution to a MOP is Pareto optimal if there exists no other feasible solution which would decrease some criteria without causing a simultaneous increase in at least one other criterion. The set comprising all of Pareto optimal solutions is just the Pareto optimal set. Generally the image set of all feasible solutions in a MOP does not constitute a totally ordered set, instead a partially ordered set. The theoretical background on the partially ordered set in this paper roots in $[6,14]$.

Let $F$ be a set., we can define a partial order relation " $\leq$ " which is a reflexive, antsymmetric and transitive relation on F, and a strict partial order relation " $<$ " as an antireflexive, asymmetric and transitive relation which may be obtained by the former relation by setting $x<y:=(x \leq y)^{\wedge}(x \neq y)$.

Definition 2.1. Let $F$ be some set. If the partial order relation " $\leq$ " is valid on $F$ then the pair $(F, \leq)$ is called a partially ordered set (or short: poset). If $x<y$ for some $x$, $y \in F$ then $x$ is said to dominate $y$. Distinct points $x, y \in F$ are said to be comparable when $x<y, y<x$ or $x=y$. Otherwise, $x$ and $y$ are incomparable which is denoted by $x$ II $y$. If each pair of distinct points of a poset $(F, \leq)$ is comparable then $(F, \leq)$ is called a totally ordered set or a chain. Dually, if each pair of distinct points of a poset $(F, \leq)$ are incomparable then $(F, \leq)$ is termed an antichain. An element $x^{*} \in F$ is called a minimal element of the poset $(F, \leq)$ if there is no $x \in F$ such that $x<x^{*}$. The set of all minimal elements, denoted $M(F, \leq)$, is said to be complete [9, 21$]$ if for each $x \in F$ there is at least one $x^{*} \in M(F, \leq)$ such that $x^{*} \leq x$.

If the poset $(F, \leq)$ is finite then the completeness of $M(F, \leq)$ is guaranteed [6]. Let $f: X \rightarrow F$ be a mapping from some set $X$ to the poset $(F, \leq)$. For some $A \subseteq X$ the set $M_{f}$ $(A, \leq)=\{a \in A: f(a) \in M(f(A), \leq)\}$ contains those elements from $A$ whose images are minimal elements in the image space $f(A)=\{f(a): a \in A\}$. In order to clarify the notion of "stochastic convergence to the set of minimal elements" we need measures on the distances between finite point sets. Here the first measure is characterized as follows: If $A$ and $B$ are subset of a finite ground set $X$ then $d(A, B)=\left|A \cup_{B \mid}-\right| A \bigcap_{B \mid}$ is a metric on the power set of $X$. the second measure uses quantity $\delta B(A)=|A|-|A \cap B|$ counting the number of elements that are in set $A$ but not in set $B$. 
Definition 2.2. Let $A_{t}$ be a solutions set of a MOA at iteration $t \geq 0$ and $F_{t}=f\left(A_{t}\right)$ its associated image set, $F^{*}$ denotes the set of minimal elements. The algorithm is said to converge with probability 1 to the entire set of minimal elements if

$$
d\left(F_{t}, F^{*}\right) \rightarrow 0 \text { with probability } 1 \text { as } t \rightarrow \infty \text {. }
$$

And the algorithm is said to converge with probability 1 to the set of minimal elements if

$$
\delta_{F^{*}}\left(F_{t}\right) \rightarrow 0 \text { with probability } 1 \text { as } t \rightarrow \infty \text {. }
$$

Needless to say, $d\left(F_{t}, F^{*}\right) \rightarrow 0$ implies $\delta_{F^{*}}\left(F_{t}\right) \rightarrow 0$.

\section{The Basic Principles and the General Framework of Quantum- Inspired Multi-Objective Evolutionary Algorithms}

A few researchers have proposed some QMOEAs that are mainly based on a particular MOEAs, such as Kim, Kim and Han's QMEA based on the NSGA2 in [13] and Meshoul, Mahdi and Batouche's algorithm based on SPEA2 in [12]. Here we present a new general QMOEA framework, which is based on the basic principles of QEA and the general schemes of MOEA.

\subsection{The Basic Principles of Quantum-Inspired Evolutionary Algorithm}

\section{A. Q-bits' Chromosome Representation and Q-individual}

The individuals' chromosomes in QEA utilize Q-bits representation which is a kind of probabilistic representation. Q-bit (or qubit) is abstraction of quantum bit. It is the smallest unit of information in QEA, which is defined with a pair of numbers $(\alpha, \beta)$ [15]. Consequently, an individual's chromosome $q$ can be defined as $\mathrm{m}$ Q-bits string

$$
q=\left(\begin{array}{c|c|c|c}
\alpha_{1} & \alpha_{2} & \ldots & \alpha_{m} \\
\beta_{1} & \beta_{2} & \ldots & \beta_{m}
\end{array}\right)
$$

where $\left|\alpha_{i}\right|^{2}+\left|\beta_{i}\right|^{2}=1, i=1,2, \ldots m$. In this paper we call this kind of individual as $Q$-individual. This quantum representation mechanism has the advantage to represent a linear superposition of states. All possible combinations of decision variables values can be derived from a single Q-individual.

\section{B. Q-population and observing population}

For more diversity, QEA maintains a population of Q-individuals, called $Q$ population in this paper, using $Q(t)=\left\{q_{1}{ }^{t}, q_{2}{ }^{t}, \ldots q_{n}{ }^{t}\right\}$ at each generation $\mathrm{t}$ of the evolutionary iterative process where $\mathrm{n}$ is the size of population and $q_{j}^{t}$ is a Qindividual defined as above text.

A quantum operator called observing is applied in order to obtain feasible solutions in QEA. This operator makes a population of binary solutions, $P(t)=\left\{x_{1}{ }^{t}, x_{2}{ }^{t}, \ldots x_{n}{ }^{t}\right\}$, which is called observing population in the present paper. Each component $x_{j}^{t}$, 
$j=1,2, \ldots n$ is a length $m$ binary string which is formed by selecting either 0 or 1 for each bit by using the probability either $\left|\alpha_{i}\right|^{2}$ or $\left|\beta_{i}\right|^{2}, k=1,2, \ldots m$ of $q_{j}^{t}$, respectively.

\section{Updating Q-individual and Q-gate}

In QEA process Q-individuals can be updated by applying a variety of Q-gate operators, by which the updated Q-bit with a new pair of number $\left(\alpha^{\prime}, \beta^{\prime}\right)$ should satisfy the normalization condition, $\left|\alpha^{\prime}\right|^{2}+\left|\beta^{\prime}\right|^{2}=1$. The rotation gate acting on a single Q-bit is the basic Q-gate in QEA as follows:

$$
R(\Delta \theta)=\left(\begin{array}{cc}
\cos (\Delta \theta) & -\sin (\Delta \theta) \\
\sin (\Delta \theta) & \cos (\Delta \theta)
\end{array}\right)
$$

where $\Delta \theta$ is a rotation angle toward either 0 or 1 state depending on its objective sign. As the rotation gate is applied, a correlative binary individual to each Q-individual, which is called an objective solution, is often appointed in advance. The objective sign to each bit of a Q-individual is defined as the corresponding bit of the correlative objective binary individual, respectively. After the rotation gate $R(\Delta \theta)$ acting on a Qbit $(\alpha, \beta)$, the updated Q-bit $\left(\alpha^{\prime}, \beta^{\prime}\right)$ satisfy $\left(\alpha^{\prime}, \beta^{\prime}\right)^{T}=R(\Delta \theta) \cdot(\alpha, \beta)^{T}$. Here $\Delta \theta$ should be designed in compliance with the application problem and each Q-bit possibly matches with different angles. Several rotation gate strategies have been given, here we use the $\Delta \theta$ strategy in [10].

Moreover NOT gate and $H_{\varepsilon}$ gate are other two operator. The function of the former is to exchange the probabilities of ' 0 ' state and ' 1 ' state in the Q-bit. It can be defined as a transformation matrix $N=\left(\begin{array}{ll}0 & 1 \\ 1 & 0\end{array}\right)$. The latter is extended from the rotation gate and was proposed by Han and Kim in [11]. If acted by $H_{\varepsilon}$ gate, a Q-bit $(\alpha, \beta)$ would be updated as $\left(\alpha^{\prime}, \beta^{\prime}\right)^{T}=H_{\varepsilon}(\alpha, \beta, \Delta \theta)$, where for $\left(\alpha^{\prime \prime}, \beta^{\prime \prime}\right)^{T}=R(\Delta \theta)(\alpha, \beta)^{T}$

a) if $\left|\alpha^{\prime \prime}\right|^{2} \leq \varepsilon$ and $\left|\beta^{\prime \prime}\right|^{2} \geq 1-\varepsilon$ then $\left(\alpha^{\prime}, \beta^{\prime}\right)^{T}=\left(\operatorname{sgn}\left(\alpha^{\prime \prime}\right) \cdot \sqrt{\varepsilon}, \operatorname{sgn}\left(\beta^{\prime \prime}\right) \cdot \sqrt{1-\varepsilon}\right)^{T}$;

b) if $\left|\alpha^{\prime \prime}\right|^{2} \geq 1-\varepsilon$ and $\left|\beta^{\prime \prime}\right|^{2} \leq \varepsilon$ then $\left(\alpha^{\prime}, \beta^{\prime}\right)^{T}=\left(\operatorname{sgn}\left(\alpha^{\prime \prime}\right) \cdot \sqrt{1-\varepsilon}, \operatorname{sgn}\left(\beta^{\prime \prime}\right) \cdot \sqrt{\varepsilon}\right)^{T}$;

c) otherwise $\left(\alpha^{\prime}, \beta^{\prime}\right)^{T}=\left(\alpha^{\prime \prime}, \beta^{\prime \prime}\right)^{T}$.

Here $0<\varepsilon<1$.

\subsection{The General Framework of Quantum-Inspired Multi-Objective Evolutionary Algorithms}

The algorithms for MOP have two main goals in the iterative process: making current solutions as close as possible to the Pareto front and as diverse as possible. A number of good techniques have been used in order to improve MOEAs, some of them are so successful that they have become general schemes, such as nondominated rank sorting and selection, maintaining solutions diversity and reserving elitism solutions as an external population etc[16]. Integrating the basic principle of QEA and general schemes of MOEA, we propose a general framework of quantum-inspired multiobjective evolutionary algorithms as follows: 
The Procedure of the QMOEAs' Basic Framework

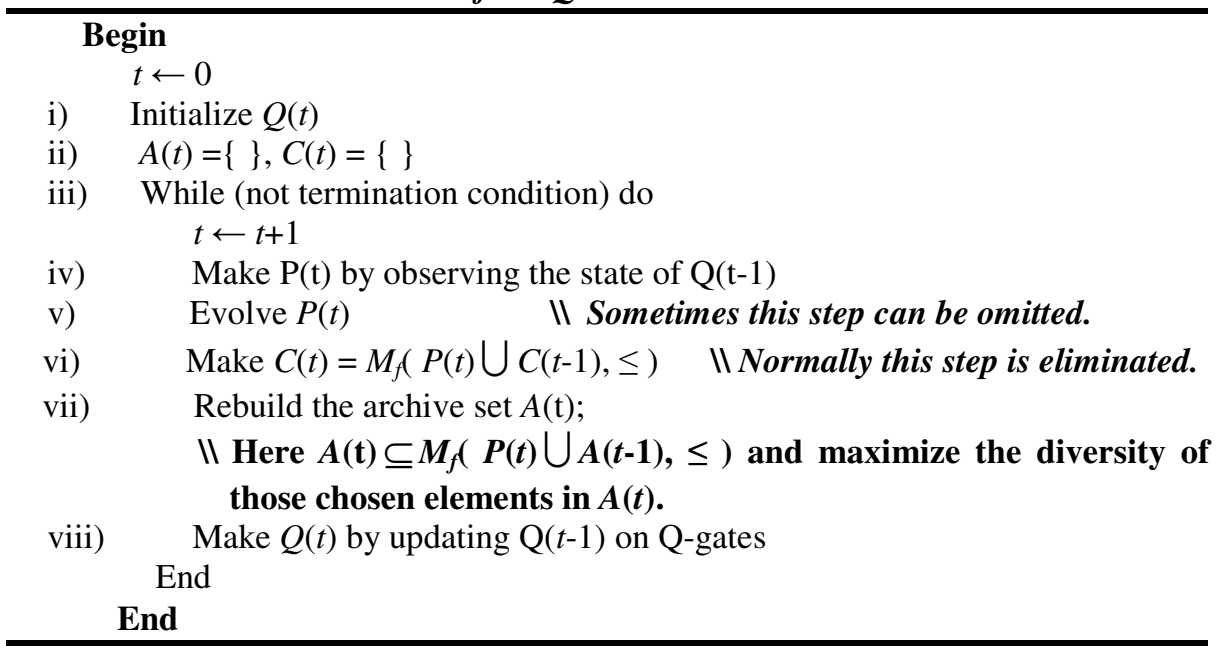

i) ii) First the two external archive set $A(t), C(t)$ and the Q-population $Q(t)$ are initialized. Set $A(0)=\phi, C(0)=\phi$. Make $Q(0)=\left\{q_{j}^{0}, j=1,2, \ldots n\right\}$, where each Q-bit in $q_{j}^{0}$ have the identical probability of ' 0 ' state and ' 1 ' state. In other word, each Q-bit of $q_{j}^{0}$ can be presented as $\left(\frac{1}{\sqrt{2}}, \frac{1}{\sqrt{2}}\right)$.

iii) Until the termination condition is satisfied, the QMOEA is running in the while loop.

iv) Binary solutions in $P(t)$ are formed by observing the state of $Q(t)$ as above subsection.

v) $\quad P(t)$ can evolve by using some evolutionary operations, such as simple genetic algorithm, evolutionary strategy etc. In fact, this step is not indispensable; it may be omitted in some QMOEAs.

vi) According to the definition of $C(t)=M_{f}(P(t) \bigcup C(t-1)), C(t)$ consists of all nondominated solutions in $\bigcup_{t_{1}=1}^{t} P\left(t_{1}\right)$. Here some efficient techniques can be used, such as the fast nondominated sorting method which was proposed in NSGA2 [4]. Since the size of $C(t)$ will continually grow along with the iteration cycles and may be too huge, this step usually is not adopted in practice.

vii) As $A(\mathrm{t}) \subseteq M_{f}(P(t) \bigcup A(t-1)$, $\leq)$, all elements of $A(t)$ are the nondominated solutions in $\bigcup_{t_{1}=1}^{t} P\left(t_{1}\right)$. Unlike $C(t), A(t)$ is the archive set, its size is usually changeless. In order to maximize its diversity, some techniques can be used such as crowding-distance [4], clustering [17] etc.

viii) In this step, Q-individuals in $Q(t)$ are updated by applying Q-gates, such as the rotation gate, NOT gate and $H_{\varepsilon}$ gate. When the rotation gate are applied, a correlative solution to each Q-individual, which is called a objective solution, is often selected from $A(t)$. Then the objective sign to each bit of a Q-individual is defined as the corresponding bit of the correlative objective solution, respectively. 


\section{On the Convergence Properties of QMOEA}

\subsection{One of the Sufficient Convergence Conditions to QMOEA}

According to those definitions of MOP and partially ordered set in section 2, we look upon the image space of MOP, $(f(S), \leq)$, as a partially ordered set. The set $M(f(S), \leq)$, a subset of $(f(S), \leq)$, denotes the Pareto optimal set of the MOP. By the construction of the basic framework, $A(t)$ is the archives solutions set. Thus we can define the concept on convergence to the Pareto optimal set as follows.

Definition 4.1. Let $F^{*}=M(f(S), \leq)$ and $A(t)$ be the archives solutions set of QMOEA. The QMOEA is said to converge with probability 1 to the Pareto optimal set if

$$
\delta_{F^{*}}(f(A(t))) \rightarrow 0 \text { with probability } 1 \text { as } t \rightarrow \infty \text {. }
$$

Proposition 1. One of sufficient conditions by whose the QMOEA converges with probability 1 to its Pareto optimal set is that the set sequence $\{C(t)\}$ satisfy

$$
d\left(f(C(t)), F^{*}\right) \rightarrow 0 \text { with probability } 1 \text { as } t \rightarrow \infty \text {. }
$$

where $F^{*}=M(f(S), \leq)$ is the minimal elements set of the image set $f(S)$.

Proof: Since $C(t)=M_{f}(P(t) \cup C(t-1)), t>0$ and $C(0)=\phi, A(t) \subseteq M_{f}(P(t) \cup A(t-1)), t>0$ and $A(0)=\phi$, we can attain $A(t) \subseteq C(t)$ and $f(A(t)) \subseteq f(C(t))$.

Let $\mathrm{S}_{0}=f(C(t))-f(A(t))$. We can conclude that $d\left(f(C(t)), F^{*}\right)=\left|f(C(t)) \cup F^{*}\right|-\left|f(C(t)) \cap F^{*}\right|$

$=|f(A(t))|-\left|f(A(t)) \cap F^{*}\right|+\left|S_{0} \cup F^{*}\right|-\left|f(C(t)) \cap F^{*}\right|$

$=\delta_{F^{*}}(f(A(t)))+\left|S_{0} \cup F^{*}\right|-\left|f(C(t)) \cap F^{*}\right|$

$\geq \delta_{F^{*}}(f(A(t)))$.

Since $\delta_{F^{*}}(f(A(t))) \leq d\left(f(C(t)), F^{*}\right)$, it is clear that if $d\left(f(C(t)), F^{*}\right) \rightarrow 0$ with probability 1 as $t \rightarrow \infty$ then $\delta_{F^{*}}(f(\mathrm{~A}(\mathrm{t}))) \rightarrow 0$ with probability 1 as $t \rightarrow \infty$. Considering the definition (4.1), we have proved this proposition.

Proposition 2. Let $S$ be a feasible solution set of MOP, s $\in S$ be an arbitrary from feasible solution. If the probability $P(s \in P(t))$ is independent each other for different $t$ and there exists a real number $\varepsilon_{0}, 0<\varepsilon_{0}<1$, which satisfies $P(s \in P(t)) \geq \varepsilon_{0}$ for all $s \in S$, all $t>0$, then $d\left(f(C(t)), F^{*}\right) \rightarrow 0$ with probability 1 as $t \rightarrow \infty$, where $F^{*}=M(f(S), \leq)$ is the minimal elements set of the image set $f(S)$.

Proof: In one 'while loop' of the basic framework the $P(t)$ maybe be changed in v) step. For avoiding the different understanding, the $P(t)$ always denotes its final result in v) step in following text.

First, we describe $d\left(f(C(t)), F^{*}\right) \rightarrow 0$ with probability 1 as $t \rightarrow \infty$ with a mathematical limit language as follows:

$\forall \varepsilon_{1} \in R, 0<\varepsilon_{1}<<1, \exists N_{0} \in N$ such that $P\left(d\left(f(C(t)), F^{*}\right)=0\right) \geq 1-\varepsilon_{1}$ for all $\mathrm{t}>N_{0}$.

Second, we consider the preconditions that can guarantee $d\left(f(C(t)), F^{*}\right)=0$. The poset $f(\mathrm{~S})$ is complete since the feasible set $S$ and its image poset $f(\mathrm{~S})$ are finite. Let $x$ be an arbitrary element of $f(\mathrm{~S})-F^{*}$. There exists at least an element $y \in F^{*}$ and $y$ dominate $x$. By the definitions of $C(t)$, It is guaranteed that if $y \in f\left(C\left(t_{0}\right)\right)$ then $x$ 
$\in f(\mathrm{C}(t))$ is impossible for all $t \geq t_{0}$. Further, if $F^{*} \subseteq f\left(C\left(t_{0}\right)\right)$ implies that any element of $f(\mathrm{~S})-F^{*}$ will not stay in $f(\mathrm{C}(t))$ for all $t \geq t_{0}$. In other words, $F^{*} \subseteq f\left(C\left(t_{0}\right)\right)$ implies $\left(f(S)-F^{*}\right) \cap f(C(t))=\phi$ for all $t \geq t_{0}$. Since $f(C(t))$ is a subset of $f(S)$, we can affirm that if $F^{*} \subseteq f\left(C\left(t_{0}\right)\right)$ and $\left(f(S)-F^{*}\right) \cap f(C(t))=\phi$ then $F^{*}=f(C(t))$. Hence, it is clear that if $F^{*} \subseteq f\left(C\left(t_{0}\right)\right)$ then $d\left(f(C(t)), F^{*}\right)=\left|f(C(t)) \cup F^{*}\right|-\left|f(C(t)) \cap F^{*}\right|=0$ for all $t \geq t_{0}$.

Third, we estimate the probability that all element of $F^{*}$ enter into $f(C(t)$ in $K \times l$ iterations beginning from $t_{0}, P\left(F^{*} \subseteq f\left(C\left(t_{0}+K \times l\right)\right)\right)$, as follows. By construction of the basic QMOEA framework and those definitions of $C(\mathrm{t})$, it is guaranteed the image set $f(C(t))$ is the minimal set of the union set $\bigcup_{t_{1}=1}^{t} f\left(P\left(t_{1}\right)\right)$. As soon as an element of $F^{*}$ has entered $f\left(P\left(t_{0}\right)\right)$ then it will be saved in $f\left(C\left(t_{0}\right)\right)$ and then it will stay in $f(C(t)), t \geq$ $t_{0}$, forever.

Let $K=\left|F^{*}\right|$. Without loss of generality, we can assume that all elements of $\left|F^{*}\right|$ are label as $\left\{s_{1}, s_{2}, \ldots, s_{K}\right\}$. Taking into account that the probability $P(s \in P(t))$ is independent each other for different $t$, we can decompose these probability expressions in following inequations. Since there exists a real number $\varepsilon_{0}, 0<\varepsilon_{0}<1$, which satisfies $P(s \in P(t)) \geq \varepsilon_{0}$ for all $s \in S$, all $t>0$, we can estimate the probability that an element $s_{\mathrm{j}}, j=1,2, \ldots, K$, enter into $f(C(t))$ in $l$ iterations beginning from $t_{0}$ as follows:

$$
\begin{aligned}
& P\left(s_{j} \in f\left(C\left(t_{0}+l\right)\right) \mid s_{j} \notin f\left(C\left(t_{0}\right)\right)\right) \\
& =1-P\left(s_{j} \notin f\left(C\left(t_{0}+l\right)\right) \mid s_{j} \notin f\left(C\left(t_{0}\right)\right)\right) \\
& =1-P\left(\left(s_{j} \notin f\left(C\left(t_{0}\right)\right)\right) \operatorname{and}\left(s_{j} \notin f\left(P\left(t_{0}+1\right)\right)\right) \operatorname{and}\left(s_{j} \notin f\left(P\left(t_{0}+2\right)\right)\right)\right. \\
& \left.\quad \text { and ...and }\left(s_{j} \notin f\left(P\left(t_{0}+l\right)\right)\right)\right) \\
& =1-P\left(s_{j} \notin f\left(C\left(t_{0}\right)\right)\right) \cdot P\left(s_{j} \notin f\left(P\left(t_{0}+1\right)\right)\right) \cdot P\left(s_{j} \notin f\left(P\left(t_{0}+2\right)\right)\right) \cdot \ldots \cdot P\left(s_{j} \notin f\left(P\left(t_{0}+l\right)\right)\right) \\
& \geq 1-\left(1-\varepsilon_{0}\right)^{l},
\end{aligned}
$$

where $l$ and $t_{0}$ are arbitrary nature number.

Further, we can estimate the probability that all element of $F^{*}$ enter into $f(C(t)$ in $K \times l$ iterations beginning from $t_{0}$ as follows:

$$
\begin{aligned}
& P\left(F^{*} \subseteq f\left(C\left(t_{0}+K \times l\right)\right)\right) \\
& \geq P\left(\left(s_{1} \in P\left(t_{0}+l\right)\right) \text { and }\left(s_{2} \in P\left(t_{0}+2 l\right)\right) \text { and } \ldots \text { and }\left(s_{K} \in P\left(t_{0}+K \times l\right)\right)\right) \\
& \left.\left.\left.\left.\geq P\left(s_{1} \in P\left(t_{0}+l\right)\right) \mid s_{1} \notin P\left(t_{0}\right)\right)\right) \cdot P\left(s_{2} \in P\left(t_{0}+2 l\right)\right) \mid s_{2} \notin P\left(t_{0}+l\right)\right)\right) . \\
& \left.\left.\quad \quad \ldots \cdot P\left(s_{K} \in P\left(t_{0}+K \times l\right)\right) \mid s_{K} \notin P\left(t_{0}+(K-1) \times l\right)\right)\right) \\
& \geq\left(1-\left(1-\varepsilon_{0}\right)^{l}\right)^{K},
\end{aligned}
$$

where $l$ and $t_{0}$ are arbitrary nature number.

Finally, we can sum up the proof by a fit $N_{0}$ for arbitrary $\varepsilon_{1}$ as follows.

Let $N_{1}=\log _{1-\varepsilon_{0}}^{1-\left(1-\varepsilon_{1}\right)^{\frac{1}{K}}} \cdot \forall \varepsilon_{1} \in R, 0<\varepsilon_{1}<<1$, we set $N_{0} \geq K \cdot N_{1}+1$ and $N_{0} \in N$. Let $t_{0}=1$ and $\mathrm{t}>N_{0}$. With all the above conclusions we can conclude as follows:

$P\left(d\left(f\left(C(t), F^{*}\right)=0\right)\right.$

$\geq P\left(F^{*} \subseteq f\left(C\left(t_{0}+K \cdot N_{1}\right)\right)\right.$ 


$$
\begin{aligned}
& \geq\left(1-\left(1-\varepsilon_{0}\right)^{N_{1}}\right)^{K} \\
& =1-\varepsilon_{1} .
\end{aligned}
$$

Summing up: $\forall \varepsilon_{0}, \varepsilon_{1} \in R, 0<\varepsilon_{0}, \varepsilon_{1}<<1, \exists N_{0} \in N$ such that $\left(1-\left(1-\varepsilon_{0}\right)^{t}\right)^{N_{1}} \geq 1-\varepsilon_{1}$ for all $t$ $>N_{0}$, i.e. it is true that $d\left(f(C(t)), F^{*}\right) \rightarrow 0$ with probability 1 as $t \rightarrow \infty$.

Considering the proposition 1 and proposition 2 , we can immediately conclude the theorem 1 as follows, whose proof is omitted.

Theorem 1 (Sufficient Convergence Condition). Let $S$ be a feasible solution set of MOP. One of the sufficient conditions by whose this QMOEA converges with probability 1 to its Pareto optimal set is that there exists a real number $\varepsilon_{0}, 0<\varepsilon_{0}<1$, which satisfies $P(s \in P(t)) \geq \varepsilon_{0}$ for all $s \in S, t>0$ and $P(s \in P(t))$ is independent from each other for different $t$.

Remark 1. From theorem 1, we obtain a sufficient convergence condition of QMOEA to the Pareto optimal set. However, it is not indispensable. We can give an example which does not satisfy this sufficient condition but converge to its Pareto optimal set.

\subsection{On the Convergence Property of QMOEA with $H_{\varepsilon}$ Gate}

The first example algorithm meeting the convergence condition is the MOEA with $H_{\varepsilon}$ gate. The $H_{\varepsilon}$ gate is firstly proposed by Kim and Han in [11] and we have simply described it in subsection (3.1). The procedure of this algorithm is similar with that basic framework but the step v) and vi) are eliminated and the $H_{\varepsilon}$ gate is adopted in step viii).

The Procedure of the $Q M O E A$ with $H_{\varepsilon}$ Gate

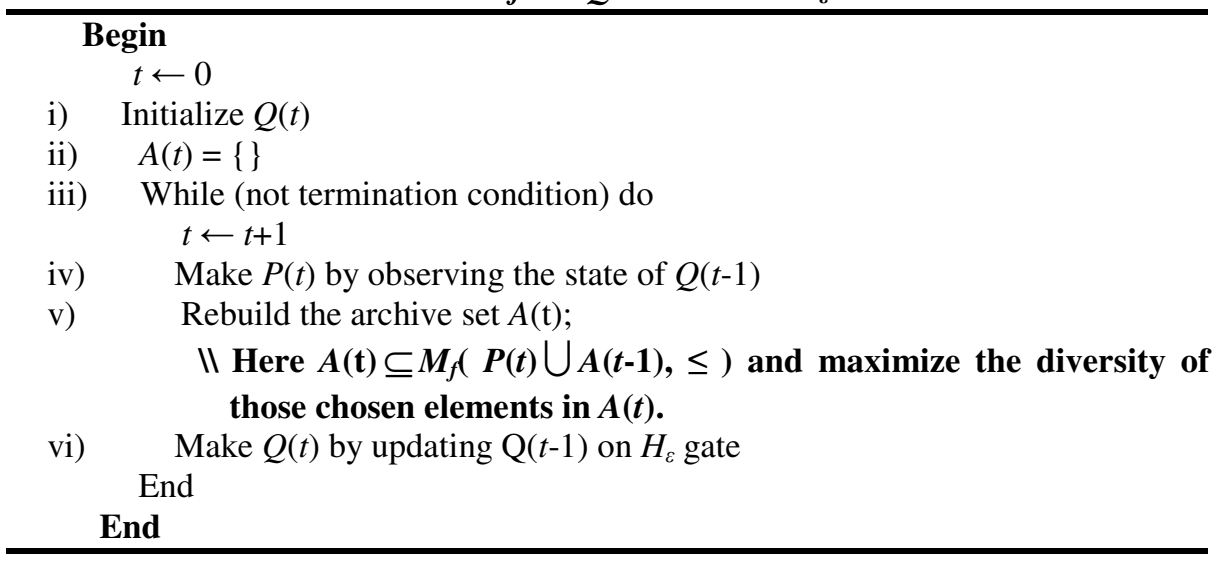

Theorem 2. The QMOEA with $H_{\varepsilon}$ gate which is defined above converges with probability 1 to its Pareto optimal set.

Proof: Taking into account the definition of $H_{\varepsilon}$ gate, we can conclude 


$$
\exists 0<\mathcal{E}<1, \forall q_{j}^{t}=\left(\begin{array}{c|c|c|c|c}
\alpha_{j 1}^{t} & \alpha_{j 2}^{t} & \ldots & \alpha_{j m}^{t} \\
\beta_{j 1}^{t} & \beta_{j 2}^{t} & \ldots & \beta_{j m}^{t}
\end{array}\right) \in Q(t), \mathcal{E} \leq\left|\alpha_{j k}^{t}\right|^{2},\left|\beta_{j k}^{t}\right|^{2} \leq 1-\mathcal{E} \text { where }
$$
$j=1,2, \ldots n, k=1,2, \ldots, m, \mathrm{t}>0,0<\varepsilon<<1$.

According to the observing operator, if we define the function $\operatorname{observing}(\alpha, \beta)$ as one observing operator to Q-bit $(\alpha, \beta)$, it can only get either 1 or 0 . We can estimate the probability of the observing result to a Q-bit $\left(\alpha_{j k}^{t}, \beta_{j k}^{t}\right)$ as follows:

$$
\begin{aligned}
& P\left(\text { observing }\left(\alpha_{j k}^{t}, \beta_{j k}^{t}\right)=0\right)=\left|\alpha_{j k}^{t}\right|^{2} \geq \varepsilon, \\
& P\left(\text { observing }\left(\alpha_{j k}^{t}, \beta_{j k}^{t}\right)=1\right)=\left|\beta_{j k}^{t}\right|^{2} \geq \varepsilon .
\end{aligned}
$$

Let us now consider the probability $P(s \in P(t)), s \in S, t>0$. On the assumption that $s$ is an arbitrary element in $S, s$ can be expressed as a binary string $\left\{s^{1} s^{2} \ldots s^{m}\right\}$, where $s^{k}$ is either 0 or $1, k=1,2, \ldots, m$. Further we can conclude the probability of the observing result to a Q-individual $q_{j}^{t}$ :

$$
\left.P\left(\operatorname{observing}\left(q_{j}^{t}\right)\right)=s\right)=\prod_{k=1}^{m} P\left(\text { observing }\left(q_{j k}^{t}\right)=s^{k}\right) \geq \varepsilon^{m}, j=1,2, . . n, t>0 \text {. Thus we }
$$

can conclude that $\left.P(s \in P(t)) \geq P\left(\operatorname{observing}\left(q_{j}^{t}\right)\right)=s\right) \geq \varepsilon^{m}$.

Moreover, Considering the construction of the algorithm, it is guaranteed that $P(s \in P(t))$ is independent each other for different $t$.

From the theorem 1, we can conclude the theorem 2.

\subsection{On the Convergence Property of QMOEA Rotation Gate and $N_{\varepsilon}$ Gate}

The second example is the MOEA with the rotation gate and the $N_{\varepsilon}$ gate. We have described the rotation gate and NOT gate in subsection (3.1). The $N_{\varepsilon}$ gate is a modified NOT gate which is proposed in this paper. In fact its function is to exchange Q-bit's parameters with the probability $\varepsilon$. Its transformation matrix can be defined as follows:

$N_{\varepsilon}: N_{\varepsilon}=\left(\begin{array}{ll}0 & 1 \\ 1 & 0\end{array}\right)$ with probability $\varepsilon ; N_{\varepsilon}=\left(\begin{array}{ll}1 & 0 \\ 0 & 1\end{array}\right)$ with probability $1-\varepsilon$, where $0<\varepsilon<<1$.

The simplified procedure of this algorithm is similar with that basic framework but the step v) and vi) are eliminated and the rotation gate and the $N_{\varepsilon}$ gate are adopted in step viii).

The Procedure of the QMOEA with Rotation Gate and NOT Gate with Probability

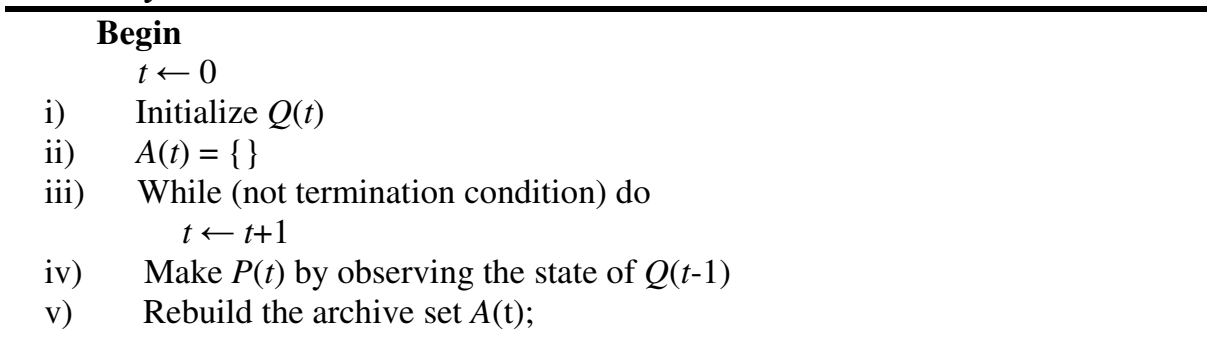


II Here $A(t) \subseteq M_{f}(P(t) \bigcup A(t-1), \leq)$ and maximize the diversity of those chosen elements in $A(t)$.

vi) Make $Q(t)$ by updating $\mathrm{Q}(t-1)$ on rotation gate

vii) Update $Q(t)$ on the $N_{\varepsilon}$ gate End

End

Theorem 3. The QMOEA with the rotation gate and the $N_{\varepsilon}$ gate which is defined above converges with probability 1 to its Pareto optimal set.

Proof: First we let the $Q(t)$ in vi) step of the algorithm above as follows:

$$
Q(t)=\left\{q_{j}^{t}, j=1,2, \ldots, n\right\} \text { and } q_{j}^{t}=\left(\begin{array}{c|c|c|c|c}
\alpha_{j 1}^{t} & \alpha_{j 2}^{t} & \ldots & \alpha_{j m}^{t} \\
\beta_{j 1}^{t} & \beta_{j 2}^{t} & \ldots & \beta_{j m}^{t}
\end{array}\right), t>0 .
$$

Then let us consider the $N_{\varepsilon}$ gate. After updated by $N_{\varepsilon}$ gate, each Q-bit in $q_{j}^{t}$ has been exchanged its parameters with the probability $\varepsilon$. According to the observing operator in iv) step of the algorithm, the probability of the observing result to $k$ th Qbit $q_{j k}{ }^{t}$ of $q_{j}^{t}$ can be expressed as follows:

$$
\begin{aligned}
& P\left(\text { observing }\left(q_{j k}^{t}\right)=0\right)=(1-\varepsilon)\left|\alpha_{j k}^{t}\right|^{2}+\varepsilon\left|\beta_{j k}^{t}\right|^{2}=(1-2 \varepsilon)\left|\alpha_{j k}^{t}\right|^{2}+\varepsilon, \\
& P\left(\text { observing }\left(q_{j k}^{t}\right)=1\right)=\varepsilon\left|\alpha_{j k}^{t}\right|^{2}+(1-\varepsilon)\left|\beta_{j k}^{t}\right|^{2}=(1-2 \varepsilon)\left|\beta_{j k}^{t}\right|^{2}+\varepsilon .
\end{aligned}
$$

Since $0<\varepsilon<<1$, we can conclude

$$
P\left(\text { observing }\left(q_{j k}^{t}\right)=0\right) \geq \varepsilon \text { and } P\left(\text { observing }\left(q_{j k}^{t}\right)=1\right) \geq \varepsilon, k=1,2, \ldots m \text {. }
$$

Let us now consider the probability $P(s \in P(t)), s \in S, t>0$. On the assumption that $s$ is an arbitrary element in $S, s$ can be expressed as a binary string $\left\{s^{1} s^{2} \ldots s^{m}\right\}$, where $s^{k}$ is either 0 or $1, k=1,2, \ldots, m$. Further we can conclude the probability of the observing result to a Q-individual $q_{j}^{t}$ :

$$
\left.P\left(\text { observing }\left(q_{j}^{t}\right)\right)=s\right)=\prod_{k=1}^{m} P\left(\text { observing }\left(q_{j k}^{t}\right)=s^{k}\right) \geq \varepsilon^{m} \quad, j=1,2, . . n, t>0 .
$$

Thus we can conclude $P(s \in P(t)) \geq P\left(\right.$ observing $\left.\left.\left(q_{j}^{t}\right)\right)=s\right) \geq \varepsilon^{m}$.

Moreover, Considering the construction of the algorithm, it is guaranteed that $P(s \in P(t))$ is independent each other for different $t$.

From the theorem 1, we can conclude the theorem 3.

\section{Conclusions}

In this article we have presented a general framework for quantum-inspired multiobjective evolutionary algorithms. Roughly speaking, this is an integration of the basic principles of quantum computing and general schemes of MOEA, such as Q-bit individual presentation, observing operator, Q-gate updating operator, external archive set, nondominated sorting, diversity preserving etc. We give one of sufficient convergence conditions for the basic framework and its proof bases on the partial set theory and probability theory. Then we present two algorithms those satisfy this convergence condition. One is with $H_{\varepsilon}$ Gate and another is with the rotation gate and NOT gate with probability. These theoretical characters may be useful for designing QMOEAs. 
Despite of these theoretical features on convergence, we need numerical results with these QMOEAs. Furthermore, efficiency and diversity are also significant to multiobjective optimization algorithms besides the convergence. These issues should be subject of future work.

\section{References}

1. Ehrgott, M. : Multicriteria Optimization. second edition ed. Springer(2005)

2. Fonseca, C. M., Flemingz, P. J.:Genetic Algorithms for Multiobjective Optimization: Formulation, Discussion and Generalization. in the Fifth International Conference on Genetic Algorithms. San Mateo: Morgan Kauffman Publishers(1993)

3. Horn, J., Nafpliotis, N., Goldberg, D.E.: A Niched Pareto Genetic Algorithm for Multiobjective Optimization. in the First IEEE Conference on Evolutionary Computation, IEEE World Congress on Computational Intelligence. Piscataway,New Jersey: IEEE Service Center(1994)

4. Deb, K., et al.: A Fast and Elitist Multiobjective Genetic Algorithm: NSGA-II. IEEE Transactions on Evolutionary Computation,Vol. 6,No. 2, (2002)182-197

5. Zitzler, E., Laumanns, M., Thiele, L.:SPEA2: Improving the Strength Pareto Evolutionary Algorithm in Technical Report. Vol. 103, (2001), Computer Engineering and Communication Networks Lab (TIK), Swiss Federal Institute of Technology (ETH) Zurich

6. Rudolph, G., Agapie, A. :Convergence Properties of Some Multi-Objective Evolutionary Algorithms. in the 2000 Congress on Evolutionary Computation (CEC 2000). Piscataway (NJ): IEEE Press(2000)

7. Rudolph, G. :Evolutionary Search under Partially Ordered Fitness Sets. in the International NAISO Congress on Information Science Innovations (ISI 2001).Millet/Sliedrecht: ICSC Academic Press(2001)

8. Hanne, T. :A Multiobjective Evolutionary Algorithm for Approximating the Efficient Set. European Journal of Operational Research, Vol. 176, (2007)1723-1734

9. Narayanan, A., Moore, M.:Quantum-inspired Genetic Algorithms. IEEE International Conference on Evolutionary Computation, Vol. 20, No. 22, (1996)61-66

10. Han, K. H., Kim, J. H.: Genetic Quantum Algorithm and its Application to Combinatorial Optimization Problem. in IEEE International Conference on Evolutionary Computation,San Diego, USA(2000)

11. Han, K. H., et al. :Parallel Quantum-inspired Genetic Algorithm for Combinatorial Optimization Problem. IEEE, (2001)1422-1429

12. Meshoul, S., Mahdi, K., Batouche, M. A.: Quantum Inspired Evolutionary Framework for Multi-objective Optimization. in Progress in Artificial Intelligence, Proceedings(2005)

13. Kim, Y., Kim, J. H., Han, K. H.: Quantum-inspired Multiobjective Evolutionary Algorithm for Multiobjective 0/1 Knapsack Problems. in 2006 IEEE Congress on Evolutionary Computation. Vancouver, Canada: IEEE Press(2006)

14. Rudolph, G.: Evolutionary Search for Minimal Elements in Partially Ordered Finite Sets. in the 7th Annual Conference on Evolutionary Programming. Berlin: Springer(1998)

15. Vedral, V. ,Plenio, M.B. :Basic of Quantum Computation. Progress in Quantum Electronics, Vol. 22, (1998)1-39

16. Coello, C.A.C.:Evolutionary Multi-objective Optimization: a Historical View of the Field. IEEE Computational Intelligence Magzine. Vol. 1,No. 1, (2006)28-36

17. Mostaghim, S., Teich, J.: The Role of $\varepsilon$-dominance In Multi Objective Particle Swarm Optimization Methods,. in the 2003 Congress on Evolutionary Computation. Canberra, Australia: IEEE Press(2003) 